\title{
Câncer Cervicouterino: Correlação entre Diagnóstico e Realização Prévia de Exame Preventivo em Serviço de Referência no Norte de Minas Gerais
}

\author{
Cervical Cancer: Correlation between Diagnosis and Previous Screening Test \\ in a Reference Center in Northern Minas Gerais \\ Cáncer de Cuello Uterino: Correlación entre Diagnóstico y Ejecución Previa de \\ Examen Preventivo en Servicio de Referencia en el Norte de Minas Gerais
}

\author{
Cláudio Henrique Rebello Gomes', Jaqueline Abadia da Silva², Jeniffer Araújo Ribeiroº, Renata Moreira Mendonça Penna²
}

\section{Resumo}

Introduçáo: $\mathrm{O}$ câncer do colo uterino, terceira neoplasia maligna feminina mais comum no mundo, é a responsável por número considerável de óbitos e representa grande motivo de preocupação dos órgãos de saúde pública, que preconizam seu rastreamento através do exame citopatológico periódico. Objetivo: Conhecer o perfil das pacientes portadoras de neoplasia maligna do colo uterino quanto à realização prévia de exame preventivo, bem como identificar as causas de não realização do mesmo. Método: Trata-se de estudo descritivo realizado em Serviço de Referência na regiáo Norte do Estado de Minas, a partir de entrevistas e revisóes de prontuários de pacientes portadoras de câncer cervicouterino atendidas em hospital de referência, entre agosto de 2008 e julho de 2009. Resultados: 71 pacientes foram entrevistadas, com média de idade de 52,5 anos, com mínima de 29 e máxima de 76 anos (desvio-padrão de $13,75)$ e a maioria $(69 \%)$ residia na zona urbana. Quanto ao tipo histológico, $97,1 \%$ das pacientes eram portadoras de carcinoma de células escamosas e 64,7\% já apresentavam estádio igual ou superior a IIb. Grande parte das pacientes questionadas $(56,3 \%)$ relatou jamais ter se submetido ao exame. Entre os motivos da não realizaçáo, 17 mulheres (42\%) relataram não saber ser necessário; 9 delas (23\%) não o julgava necessário por estarem assintomáticas; 8 pacientes (20\%) sentiam vergonha do procedimento e 6 tinham dificuldades de acesso (15\%). Conclusão: A falta de informação a respeito da importância do rastreamento, entre outros aspectos, demonstrou ser fator de grande relevância na não realização do exame.

Palavras-chave: Neoplasias do Colo do Útero/diagnóstico; Prevenção de Câncer de Colo Uterino; Programas de Rastreamento; Perfil de Saúde; Esfregaço Vaginal; Epidemiologia Descritiva

Fundação Dilson de Quadros Godinho. Montes Claros (MG), Brasil. E-mail: hospital@hospitaldilsongodinho.org.br ${ }^{1}$ Cirurgião Oncológico. Presidente da Sociedade Brasileira de Cirurgia Oncológica - Capítulo Minas Gerais (MG), Brasil.

${ }^{2}$ Acadêmicos do Curso Médico da Universidade Estadual de Montes Claros (Unimontes). Montes Claros (MG), Brasil.

Endereço para correspondência: Jaqueline Abadia da Silva. Rua Raul Correa, 172. Apto. 201. Cândida Câmara. Montes Claros (MG), Brasil. CEP: $39401-029$.

E-mail: jaquemed@hotmail.com 


\section{INTRODUÇÃO}

A neoplasia maligna do colo uterino é a terceira em incidência entre as femininas e responsável por cerca de 275 mil óbitos/ano no mundo, segundo dados de $2008^{1-2}$, sendo alvo de grande preocupação por parte dos órgãos de saúde pública. É duas vezes mais frequente em países subdesenvolvidos devido à presença de fatores de risco como a frequência aumentada de exposiçáo ao Papilomavírus humano (HPV) pela iniciação sexual precoce e multiplicidade de parceiros sexuais, além de outros como multiparidade, tabagismo, hábitos de higiene precários, subnutrição e acesso dificultoso aos serviços de saúde ${ }^{3-4}$.

O exame de prevenção do câncer do colo uterino, cujos primórdios no Brasil datam da década de 1940, foi implantado na rede pública em 1999 e compóe atualmente a Política Nacional de Atenção Oncológica, sob responsabilidade do Instituto Nacional do Câncer José Alencar Gomes da Silva (INCA). Sua finalidade consiste na detecção precoce da neoplasia invasora e suas lesóes precursoras por meio da análise citológica periódica do esfregaço obtido pela coleta utilizando a técnica de Papanicolaou. Dados estatísticos revelam que o rastreamento efetivo consegue reduzir a incidência de formas invasoras do câncer de colo em até $91 \%{ }^{3}$. Recomenda-se a realizaçáo do exame a partir de 25 anos para as mulheres que já iniciaram a atividade sexual, podendo ser preterido a partir dos 65 anos, caso dois exames consecutivos tenham apresentado resultado negativo nos últimos 5 anos $^{5}$. O Brasil, apesar dos constantes esforços em educação da população e de disponibilizar o exame pela rede pública, continua apresentando taxas de incidência e mortalidade crescentes, contrapondo-se à tendência de regressão apresentada em países desenvolvidos. Pesquisa domiciliar realizada pelo Ministério da Saúde entre os anos de 2002 e 2003 em 15 capitais e Distrito Federal avaliou a relaçáo entre comportamento de risco e morbidade referida de doenças não transmissíveis no país. A partir da análise dos dados, concluiu-se que, a despeito da cobertura considerável do exame preventivo, as taxas de mortalidade não apresentaram queda como esperado ${ }^{6}$. O número de novos casos previstos para 2012 é de $17.540^{1}$. Parte daí a relevância de estudos epidemiológicos capazes de fornecer dados representativos da realidade local e que auxiliem no delineamento de novas e mais eficientes estratégias de enfrentamento ao problema.

Dessa forma, o estudo objetivou conhecer o perfil das pacientes portadoras de neoplasia maligna do colo uterino tratadas em Hospital de Referência da regiáo Norte de Minas Gerais quanto à realização prévia de exame preventivo, bem como identificar as causas da não realização do mesmo.

\section{MÉTODO}

Foi realizado estudo quantitativo, descritivo, entre agosto de 2008 e julho de 2009, com pacientes diagnosticadas com neoplasia invasora do colo uterino encaminhadas da rede pública para o Serviço de Oncologia Ginecológica do Hospital Dílson Godinho, credenciado como Unidade de Assistência de Alta Complexidade em Oncologia (UNACON) e que é referência para esse tipo de atendimento no Norte de Minas Gerais. As pacientes foram entrevistadas, informadas sobre a pesquisa e a autorizaram formalmente. Foi analisado o resultado histopatológico, sua procedência, o profissional que o realizou e a adequabilidade técnica da amostra. A proposta do levantamento foi aprovada pelo Comitê de Ética da Instituição sob protocolo número 0007/2009.

\section{RESULTADOS}

Foram entrevistadas 71 pacientes durante o período proposto. A idade variou de 29 a 76 anos, com média de $52,5$ anos (desvio-padrão 13,75$)$ e a maioria $(69 \%)$ residia em zona urbana. Quanto ao nível de escolaridade, 40 possuíam ensino fundamental incompleto (56,3\%), 17 eram analfabetas (42,5\%), 6 possuíam ensino fundamental incompleto $(8,4 \%), 4$ ensino médio incompleto e 4 informaram ensino médio completo $(5,6 \%)$.

Em relação ao tipo histológico, o carcinoma de células escamosas predominou, sendo encontrado em $97,1 \%$ dos laudos, seguido do adenocarcinoma. Quanto ao estágio, 25 pacientes (35\%) apresentavam estadiamento de Ia2 até IIa, enquanto na maioria (46 pacientes) o estadiamento era igual ou superior a IIb, como demonstra o Gráfico 1.

Quanto ao tratamento, 43 mulheres $(60,5 \%)$ realizaram radio e quimioterapia primárias, enquanto as demais foram inicialmente submetidas a procedimento cirúrgico. Desse total de mulheres tratadas, $20(28,1 \%)$ foram reabordadas posteriormente, sendo 12 delas tratadas com radio e quimioterapia adjuvantes e $o$ restante com cirurgias de resgate. Questionadas sobre o motivo que as levou a procurar atendimento médico e obter o diagnóstico, 46 (64,7\%) referiram sangramentos

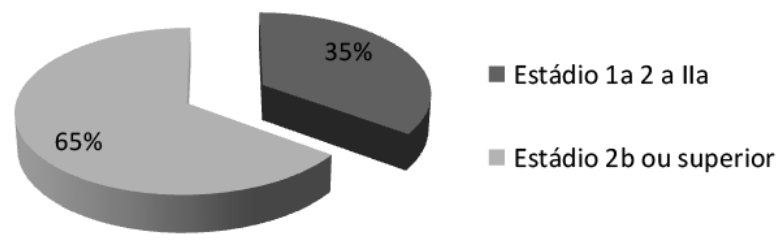

Gráfico 1. Proporção de mulheres em relação ao estadiamento no diagnóstico 
anormais, $13(18,3 \%)$ obtiveram o diagnóstico em consulta de rotina e $12(16,9 \%)$ referiram dor.

Em relação ao exame de prevenção, a maioria $(56,3 \%)$ relatou nunca havê-lo feito antes do diagnóstico, como nos mostra o Gráfico 2. Desse total, 17 (42,5\%) justificaram a não realização por não saberem ser necessário, nove $(22,5 \%)$ receberam orientaçóes, mas julgavam-no desnecessário por não sentirem nada, oito (20\%) disseram sentir vergonha do procedimento e seis (15\%) habitavam localidades nas quais o exame não estava disponível (Gráfico 3).

Das 31 pacientes que disseram se submeter ao exame, $21(67,7 \%)$ informaram frequência anual, enquanto as demais referiram intervalos variáveis, porém todos maiores que 1 ano. Em relação à coleta, 18 delas (58\%) afirmaram ter sido feita por enfermeiros ou técnicos de enfermagem, em detrimento do restante atendido por médicos.

Questionadas a respeito de como foram informadas sobre a importância do exame no rastreamento do câncer de colo, do total de entrevistadas, $24(33,8 \%)$ revelaram nunca terem sido informadas, $20(28,1 \%)$ souberam através de campanhas em Unidades de Saúde ou por meio dos agentes comunitários, $15(21,1 \%)$ por meio de familiares ou conhecidos, nove $(12,6 \%)$ através dos médicos e três $(4,2 \%)$ em meios de comunicação.

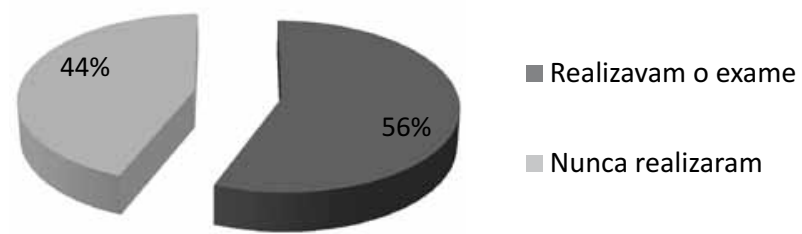

Gráfico 2. Realização prévia do exame preventivo do câncer do colo uterino

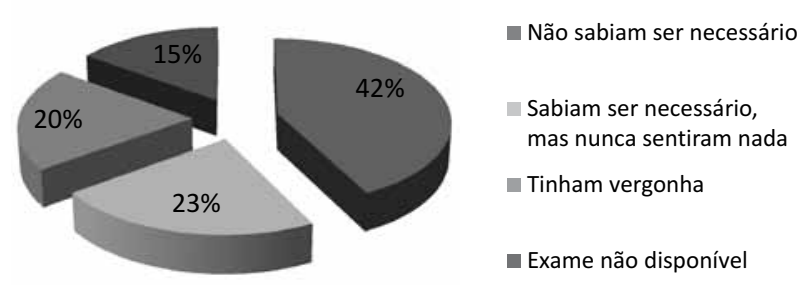

Grátıco 3. Motivos da nāo realizaçāo do exame preventivo do câncer do colo uterino

\section{DISCUSSÃO}

A maior parte das mulheres desse grupo diagnosticada com câncer do colo é proveniente da zona urbana, e está provavelmente coberta por Unidades de Atenção Básica ou fisicamente próximas dos centros de saúde. De acordo com a política atual de saúde, a Atenção Básica reveste-se de importância ímpar nos programas de rastreamento, como porta de entrada preferencial do sistema e interface entre os órgãos governamentais e a populaçãa ${ }^{1,7-9}$.

No entanto, com o fomento da Estratégia Saúde da Família no Norte de Minas na última década, acreditava-se que a maioria dos novos casos responderia primordialmente por áreas de hiatos assistenciais, predominando sobretudo nas regióes rurais ainda de acesso remoto. Mesmo frente à indisponibilidade dos dados anteriores à implementação da estratégia - que poderiam apontar para um quadro de melhora - a persistência de taxas elevadas demonstra que a expectativa não foi atendida e que há ainda muito a fazer no sentido de otimizar o alcance e eficácia do rastreamento.

No tocante à escolaridade, nota-se que o baixo nível educacional tem influência nos resultados insatisfatórios, à medida que pode determinar menor nível de informação e entendimento resultando em baixa adesão às estratégias de prevenção. A importância da informação no processo demonstrou-se a partir da constataçáo de que a maioria das mulheres que náo se submeteu ao exame preventivo não o fez por desconhecimento da necessidade do mesmo e dos aspectos ligados à doença. Estudos realizados nas regióes Nordeste e Sul do Brasil, avaliando respectivamente a percepção das mulheres acerca do exame e as causas de sua não realização, encontraram resultados semelhantes concluindo que a baixa escolaridade é fator determinante na omissão de tais mulheres na realização da prevenção ${ }^{10-11}$. Estudo paraguaio, que relacionou estratégias de prevenção do câncer à Atenção Primária, vai mais além ao enumerar a baixa escolaridade e a deficiência de informaçóes como fatores de risco diretos para a ocorrência da doença ${ }^{12}$.

É compreensível a ocorrência de grande número de casos em estadiamento avançado, uma vez que mais da metade das pacientes relatou nunca ter realizado o exame de prevenção anteriormente e procurou assistência apenas a partir do surgimento de sintomas específicos, que são via de regra indicativos de doença avançada. Dados do INCA obtidos a partir dos Registros Hospitalares de Câncer apontam para taxas de detecção em estágios avançados que chegam a $50 \%{ }^{13}$. O universo de pacientes que náo têm ainda neoplasia e que não possui orientaçóes ou estímulo à procura espontânea da prevençáo ofertada pelo sistema público é desconhecido, em grande parte devido à inexistência de registros populacionais fidedignos a partir dos quais seria possível determinar a população-alvo e, a partir daí, pensar em novas estratégias de alcance 5 .

Outro dado preocupante foi levantado por um dos autores do presente trabalho em publicação anterior, com relação ao grau de conhecimento dos médicos recém-formados nessa região para o reconhecimento de formas iniciais de neoplasias e incentivo à prevençáo por 
demanda espontânea ${ }^{14}$. No questionário semiestruturado, $74,2 \%$ dos estudantes de medicina do último período de uma universidade pública informaram que estimulavam a realização do exame para todas as pacientes, em qualquer consulta, independente do motivo. Entretanto, no presente estudo, considerando as pacientes que realizavam regularmente a prevenção, parcela significativa relatou que não recebeu orientação médica. Cabe aqui uma reflexão a respeito da importância do papel do médico da Atençáo Básica como formador de opiniáo por meio da difusão de conhecimento a respeito da doença e sua prevenção, embora o contato das pacientes durante a coleta seja em sua maioria com profissionais não médicos, como revelou o estudo.

\section{CONCLUSÃO}

A realização do presente estudo serviu aos objetivos propostos ao traçar um perfil epidemiológico local e identificar os principais fatores determinantes da relaçáo entre a não realização do exame preventivo do câncer de colo uterino e a ocorrência da doença. Conclui-se que, a despeito das eficientes estratégias e tecnologias empregadas nos programas de prevenção, estas esbarram principalmente na falta de informação, culminando com diagnósticos tardios e aumento da mortalidade. Estratégias que envolvam educação da população e difusão de conhecimentos, especialmente envolvendo a Atenção Básica, podem mostrar-se custo-efetivas em se tratando dessa preocupante lacuna na saúde brasileira que, no entanto, pode ser substancialmente diminuída.

\section{CONTRIBUIÇÕES}

Cláudio Henrique Rebello Gomes contribuiu na concepçáo e planejamento; redação e revisão crítica. Jaqueline Abadia da Silva contribuiu na obtenção e/ou análise e interpretação dos dados; redação e revisão crítica. Jeniffer Araújo Ribeiro e Renata Moreira Mendonça Penna contribuíram na obtenção e/ou análise e interpretação dos dados.

\section{Declaraçáo de Conflito de Interesses: Nada a Declarar.}

\section{REFERÊNCIAS}

1. Instituto Nacional de Câncer José Alencar Gomes da Silva. Estimativa 2012: incidência de câncer no Brasil. Rio de Janeiro: INCA; 2011. 118 p.
2. Pioli ER, Oliveira NM, Rezende AG. Caracterização da demanda de pacientes com carcinoma de colo uterino no Hospital das Clínicas da Universidade Federal de Uberlândia, Minas Gerais, Brasil, 1984-1988. Cad Saúde Pública. 1993;9(4):421-7.

3. Andrade JM. Rastreamento, diagnóstico e tratamento do carcinoma do colo do útero. [São Paulo]; 2001. 18 p. Projeto Diretrizes [da] Associação Médica Brasileira e Conselho Federal de Medicina.

4. Instituto Nacional de Câncer (Brasil). Programa nacional de combate ao câncer cérvico uterino e mama [Internet]. [citado 2005 jun 28]. Disponível em: http:www.inca.gov.br

5. Instituto Nacional de Câncer (Brasil). Diretrizes brasileiras para o rastreamento do câncer do colo do útero. Rio de Janeiro: INCA; 2011. 104 p.

6. Instituto Nacional de Câncer (Brasil). Inquérito domiciliar sobre comportamentos de risco e morbidade referida de doenças e agravos não transmissíveis: Brasil, 15 capitais e Distrito Federal, 2002-2003. Rio de Janeiro: INCA; 2004. 183 p.

7. Bottari CMS. Câncer cérvico-uterino como condição marcadora: uma proposta de avaliação da Atenção Básica [dissertação]. Rio de Janeiro: Fundação Oswaldo Cruz, Escola Nacional de Saúde Pública Sergio Arouca; 2007.

8. Oliveira MM, Pinto IC. Percepção das usuárias sobre as ações de prevenção do câncer do colo do útero na Estratégia Saúde da Família em uma distrital de saúde do município de Ribeirão Preto, São Paulo, Brasil. Rev bras saúde matern infant. 2007;7(1):31-8.

9. Cruz LMB, Loureiro RP. A comunicação na abordagem preventiva do câncer do colo do útero: importância das influências histórico-culturais e da sexualidade feminina na adesão às campanhas. Saúde Soc. 2008;17(2):120-31 .

10. Garcia CL, Pereira HC, Barreto Marinho MNAS. Percepções das mulheres acerca do exame de prevenção do câncer cérvico-uterino. Rev bras promoç saúde (Impr). 2010;23(2):118-25.

11. Thum M, Heck RM, Soares MC, Deprá AS. Câncer de colo uterino: percepção das mulheres sobre prevenção. Ciênc cuid saúde. 2008;7(4):509-16.

12. Kasamatsu E, Páez M. Cáncer de cuello uterino y virus del papiloma humano en Paraguay: perspectivas para la prevención primaria. Mem Inst Invest Cienc Salud (Impr). 2006;4(2):58-63.

13. Instituto Nacional de Câncer (Brasil). Neoplasia intra-epitelial cervical - NIC. Rev bras cancerol. 2000;46(4):355-7. Condutas do INCA/MS.

14. Gomes CHR, Silva PV, Mota FF. Comunicação do diagnóstico de câncer: análise do comportamento médico. Rev bras cancerol. 2009;55(2):139-43. 


\section{Abstract}

Introduction: Cervical cancer, the third most common female malignancy in world, is responsible for a considerable number of deaths and represents a major concern of public health agencies, who recommend its screening through regular Pap smears. Objective: To know the profile of patients with malignant cervical neoplasm as to previous screening and to identify causes for not undergoing it. Method: This descriptive study was conducted in a reference center in northern Minas Gerais through interviews and reviews of medical records of patients with cervical cancer treated at a referral hospital between August 2008 and July 2009. Results: 71 patients were interviewed, with an average age of 52.5 years with minimum 29 and maximum 76 years of age (standard deviation of 13.75) and most of them (69\%) lived in urban areas. As to the histological type, $97.1 \%$ of the patients had squamous cell carcinoma and $64.7 \%$ had at least stage IIb or above. Most of the interviewees (56.3\%) reported never having undergone the test. Among the reasons for not doing it, 17 women (42\%) reported not knowing whether it is important, 9 (23\%) considered it not necessary because they were asymptomatic, 8 patients $(20 \%)$ were ashamed of the procedure and 6 (15\%) had difficulty of access. Conclusion: The lack of information about the importance of screening, among other things, proved to be a highly relevant factor for not undergoing the examination.

Key words: Uterine Cervical Neoplasms/diagnosis; Cervix Neoplasms Prevention; Mass Screening; Health Profile; Vaginal Smears; Epidemiology, Descriptive

\section{Resumen}

Introducción: El cáncer de cuello uterino, tercera neoplasia maligna femenina más común en el mundo, es la responsable de un número considerable de óbitos y representa un gran motivo de preocupación de los órganos de salud pública, que preconizan su rastreo a través del examen citopatológico periódico. Objetivo: Conocer el perfil de las pacientes portadoras de neoplasia maligna de cuello uterino en cuanto a la realización previa de examen preventivo, así como identificar las causas de la no ejecución del examen. Método: Este es un estudio descriptivo llevado a cabo en servicio de referencia en la región norte del estado de Minas, a partir de entrevistas y revisiones de expedientes médicos de pacientes portadoras de cáncer de cuello uterino atendidas en hospital de referencia entre agosto de 2008 y julio de 2009. Resultados: 71 pacientes fueron entrevistadas, de franja etaria de 52,5 ańos, siendo la mínima de 29 y máxima de 76 años (desvío normalizado de 13,752) y la mayoría (69\%) vivía en la zona urbana. En cuanto al tipo histológico, $97,1 \%$ de las pacientes eran portadoras de carcinoma de células escamosas y $64,7 \%$ ya presentaban etapa igual o superior a IIb. Gran parte de las pacientes cuestionadas $(56,3 \%)$ relató jamás haber sido sometida al examen. Entre los motivos de la no realización 17 mujeres (42\%) relataron que no sabían si el examen era necesario o no, 9 de ellas (23\%) no lo juzgaba necesario porque no presentaban síntomas de la enfermedad, 8 pacientes (20 \%) sentían vergüenza en cuanto al procedimiento y 6 tenían dificultades de acceso (15\%). Conclusión: La falta de información acerca de la importancia del rastreo, entre otros aspectos, demostró ser un factor de gran relevancia para la no realización del examen.

Palabras clave: Neoplasias del Cuello Uterino/diagnóstico; Prevención de Cáncer de Cuello Uterino; Tamizaje Masivo; Perfil de Salud; Frotis Vaginal; Epidemiología Descriptiva 